\title{
Melatonin modulates the expression of Bcl-2 family proteins in liver after thermal injury in rats
}

\author{
Ganka Bekyarova $^{1 *}$, Maria Tzaneva ${ }^{2}$, Minka Hristova ${ }^{1}$ \\ ${ }^{1}$ Division of Pathophysiology, Medical University of Varna, Varna, Bulgaria \\ ${ }^{2}$ Department of General and Clinical Pathology, Medical University of Varna, Varna, Bulgaria \\ Email: "gabekyarova2001@yahoo.com
}

Received 26 September 2013; revised 28 October 2013; accepted 9 November 2013

Copyright (C) 2013 Ganka Bekyarova et al. This is an open access article distributed under the Creative Commons Attribution License, which permits unrestricted use, distribution, and reproduction in any medium, provided the original work is properly cited.

\begin{abstract}
Melatonin, the principal secretory product of the pineal gland, functions as a potent antioxidant and free radical scavenger. Additionally, the antiapoptotic effect of melatonin has been observed both in vivo and in vitro. The aim of this study was to investigate the protective effects of melatonin against burn-induced injury in rat liver and whether these changes were associated with oxidative stress and changes in the expression of apoptosis related genes $\mathrm{Bcl}-2$ and Bax. Melatonin (10 mg/kg, i.p.) was applied immediately after $30 \%$ of total body surface area (TBSA) burns of male Wistar rats. Malondialdehyde (MDA) as marker of oxidative stress and tumor necrosis factor (TNF- $\alpha$ ) as inflammatory marker were assayed by biochemical methods. The hepatic apoptosis related genes Bcl-2 and Bax using light immunochistochemistry were investigated, too. Hepatic TNF- $\alpha$ and MDA levels were increased significantly following severe burn. Thermal trauma increased the Bax expression without any changes of anti-apoptotic Bcl-2 protein in sinusoidal endothelial cells (SECs) of burn-treated animals compared with the control group animals as well as elevated ratio $\mathrm{Bax} / \mathrm{Bcl}-2$ suggesting the susceptibility of these cells to apoptosis. Melatonin significantly decreased the MDA and TNF- $\alpha$ levels in the liver tissue. It decreased also expression of Bax, increased expression of $\mathrm{Bcl}-2$ and reduced $\mathrm{Bax} / \mathrm{Bcl}-2$ ratio. In conclusion, experimental data show that melatonin modulates the expression of Bcl-2 family proteins by increasing anti-apoptotic Bcl-2, inhibits apoptosis and restricts the burn-induced damage.
\end{abstract}

Keywords: Melatonin; Liver; Bcl-2; Bax; Tumor Necrosis Factor; Lipid Peroxidation; Thermal Skin Injury

${ }^{*}$ Corresponding author.

\section{INTRODUCTION}

Severe burn is still a serious clinical problem in emergency medicine causing damages to organs distant from burn wound. Clinical studies have shown that liver dysfunction and damage are essential for survival in critically ill and severely burned patients $[1,2]$. Various cellular and molecular interactions such as neutrophil and macrophage activation, mitochondrial dysfunction oxygen radical, cytokine overproduction, depletion of glutathione, may be involved in the processes [3-6]. These complex mechanisms cause liver dysfunction, alteration of hepatic parenchyma and eventually cell death (apoptosis).

The increased apoptosis in liver and other organs is a result of "systemic apoptotic response" after burns [7]. Pathophysiology of burn-induced liver injury includes many mechanisms and is not entirely defined yet. The possible mechanisms for increased apoptosis are a decreased perfusion and elevation of pro-inflammatory cytokines early post burn. Oxidative stress is recognized as an important mechanism of apoptosis of hepatocytes in liver injury $[8,9]$. The increased ROS production results in an imbalance between anti-apoptotic Bcl-2 and pro-apoptotic Bax proteins which contributes to the susceptibility of the cell to apoptosis. Bax and Bcl-2 proteins are among the most commonly used markers for apoptosis [10]. Literature data about the role of free radicals in liver apoptosis after burns are scarce.

Melatonin (N-acetyl-5-methoxytryptamine) is an indoleamine endogenously produced in the pineal gland and extrapineal tissues, liver including [11-13]. Melatonin possesses a wide variety of biologic effects such as sedative, anxiolytic, antinociceptive. Melatonin plays a direct role in mitochondrial homeostasis by scavenging reactive oxygen species (ROS) and reactive nitrogen species (RNS), improving mitochondrial respiration, increasing the level of ATP synthesis, and reducing the 
harmful decrease in the mitochondrial membrane potential that can trigger the apoptotic cascade $[14,15]$. Melatonin is widely used to prevent oxidative stress induced by ischemia/reperfusion $(\mathrm{I} / \mathrm{R})$ in the liver $[9,16,17]$ as well as other organs such as gastric mucosa [10].

The aim of this experimental study was to investigate the protective effects of melatonin against burn-induced injury and whether these changes were associated with oxidative stress and changes in the expression of apoptosis-related proteins $\mathrm{Bcl}-2$ and $\mathrm{Bax}$ in rat liver.

\section{MATERIAL AND METHODS}

\subsection{Animals}

The experimental procedure was approved by the Home Office for Care and Use of Laboratory Animals and performed with a strong consideration for ethics of animal experimentation. Age-matched male rats weighing between 220 and $250 \mathrm{~g}$ fasted for $12 \mathrm{~h}$ were allowed free access to water before injury. Animals were housed in a $20^{\circ} \mathrm{C}$ and offered rat chow and water ad libitum. They were kept in dark: light cycles $(\mathrm{DL}=12: 12 \mathrm{~h})$ in individual wire-bottomed cages. Thus, lights were turned off at 8:00 p.m. and turned on at 8:00 a.m. for achieving satisfactory photoperiod.

\subsection{Thermal Injury and Melatonin Treatment}

After light ether inhalation, general anesthesia was performed using thiopental $(30 \mathrm{mg} / \mathrm{kg}$ i.p.). In order to accomplish $30 \%$ of third degree burn hot boiling water $\left(90^{\circ} \mathrm{C}\right)$ was applied on the back of the animals during a period of $10 \mathrm{sec}$. For those rats which were subjected to burn injury $4 \mathrm{~mL}$ of physiological saline was applied intraperitoneally (i.p) for immediate resuscitation following burn injury. No animals died within the first $24 \mathrm{~h}$ of post-burn period. Twenty-four male Wistar rats were divided into three equal $(\mathrm{n}=8)$ groups: 1 . control-nonburned, non-treated group (C); 2. burned, nontreated (B), 3 . burned rats, melatonin-treated $(\mathrm{B}+\mathrm{Mel})$. All the animals were given buprenorphine $(0.3 \mathrm{mg} / \mathrm{kg}$ i.p. b.w. $)$ twice daily for pain control post burn. They were reanesthetized with thiopental and sacrificed $24 \mathrm{~h}$ after burns as liver was sampled.

\subsection{Biochemical Analysis}

Livers was gently separated from the underlying tissue and homogenized in 1:5 w/v $50 \mathrm{mM}$ phosphate buffer (pH 7.4) containing $0.1 \mathrm{mM}$ EDTA at $4000 \mathrm{rpm}$ for 10 $\mathrm{min}$. The homogenate was centrifuged at $800 \times \mathrm{g}$ rpm for 15 min to discard the sediment and supernatant was frozen until analysis. All the manipulations were performed at $4^{\circ} \mathrm{C}-8^{\circ} \mathrm{C}$. Analysis was carried out immediately after thawing of the samples. For apoptosis and immunohistochemical analysis tissue samples were fixed in $10 \%$ neutral buffered formalin and embedded in paraffin.

Membrane lipid peroxidation was assayed by MDA measured by its thiobarbituric acid (TBA) reactivity of liver homogenate using the method of Porter et al. (1976) [18]. Results were expressed as $\mathrm{nmol} \mathrm{MDA} / \mathrm{g}$ tissue. They were determined using the extinction co-efficient of MDA-TBA complex at $532 \mathrm{~nm}=1.56 \times 10^{-5}$ $\mathrm{cm}^{-1} \cdot \mathrm{M}^{-1}$ solution.

Hepatic tumor necrosis factor (TNF)- $\alpha$ level was quantified using enzyme-linked immunosorbent assay (ELISA) kit (Diaclone Gen-Probe, France) specific for the previously mentioned rat cytokines according to the manufacturer's instructions and guidelines. Hepatic TNF- $\alpha$ level was expressed as $\mathrm{pg} / \mathrm{mL}$.

\subsection{Immunohistochemical Examination for Bcl-2 and Bax in Liver}

Immunohistochemical staining was performed with rabbit anti-rat Bcl-2 or Bax on paraffin sections according to the manufacturer's instructions (Santa Cruz).

Morphometric method was used to assess quantitatively enzyme contents of Bax and Bcl-2. Enzyme content was determined as: strong, score 3; moderate, score 2; weak, score 1, and lacking, score 0 on the basis of the occurrence of immunodeposits (Tzaneva, 2001) [19]. Bax and Bcl-2 contents of the liver cells were defined as the enzyme content of each cell was multiplied by their scoring factors and divided by total number of cells. Morphometric investigation was performed on 50 cells from each sample.

\subsection{Histopathological Examinations}

Tissue specimens were fixed in $10 \%$ buffered formalin $(\mathrm{pH} 7.2)$, dehydrated in ascending series of ethyl alcohol $(70 \%-100 \%)$, cleared in methyl benzoate and embedded in paraffin wax. Tissue sections of $5 \mu \mathrm{m}$ were stained with hematoxylin and eosin (H\&E) and examined using light microscope (Olympus BH-2, Tokyo, Japan). Apoptotic SECs were examined by light microscopy at a magnification of $400 \times$ (high power field). Approximately 500 SECs were examined in each high power field. The apoptotic index was defined as the number of stained cells per high power field.

\section{Statistics}

Our data were log-transformed to satisfy the assumptions required to perform parametric tests and, therefore, presented as geometric mean and $95 \%$ confidence intervals of the mean. Orthogonal contrasts in ANOVA were used to statistically analyze the difference between any two specified groups. 


\section{RESULTS}

\subsection{Changes in the Hepatic Malondialdehyde and Hepatic TNF Levels}

The hepatic MDA level was found to be higher by $48 \%$ $(\mathrm{p}<0.05)$ in the burned group than that of the control group (Figure 1). Treatment with melatonin significantly reduced the elevation in mucosal MDA level, maintaining the level close to the control values.

The plasma TNF- $\alpha$ level increased by $79 \%(\mathrm{p}<0.001)$ in the burned group when compared to those of the control one. When the rats were treated with melatonin, the concentration of hepatic TNF- $\alpha$ decreased by $21 \%$ significantly, reaching the control values at the $24^{\text {th }}$ hour after burns (Figure 2).

\subsection{Changes in the Expression on Apoptosis-Related Bcl-2 in Liver}

The expression of Bcl-2 was detected in sinusoidal endothelial cells (SECs) in the control group (Figure 3). The mean Bcl-2 content in the cells was $1.36 \pm 0.48$ (Figure 3). In the burned group, Bcl-2 expression remained low $(1.14 \pm 0.69)$ and did not show significant changes compared to controls. The expression Bcl-2 was

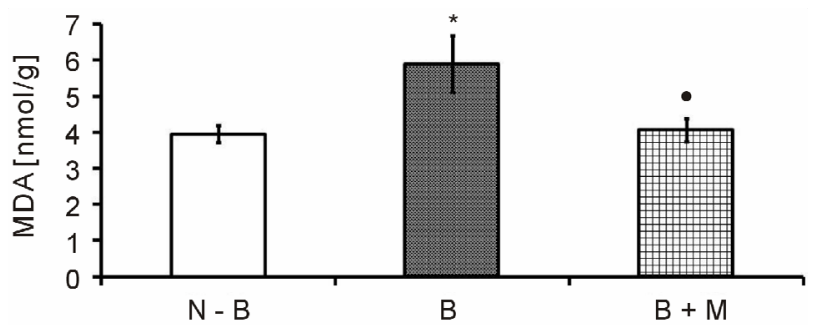

Figure 1. Effect of melatonin on burn-induced hepatic MDA level. Results are given as mean \pm SEM; ${ }^{*} \mathrm{p}<0.05$ compared with control group; $\bullet p<0.05$ compared with burned group.

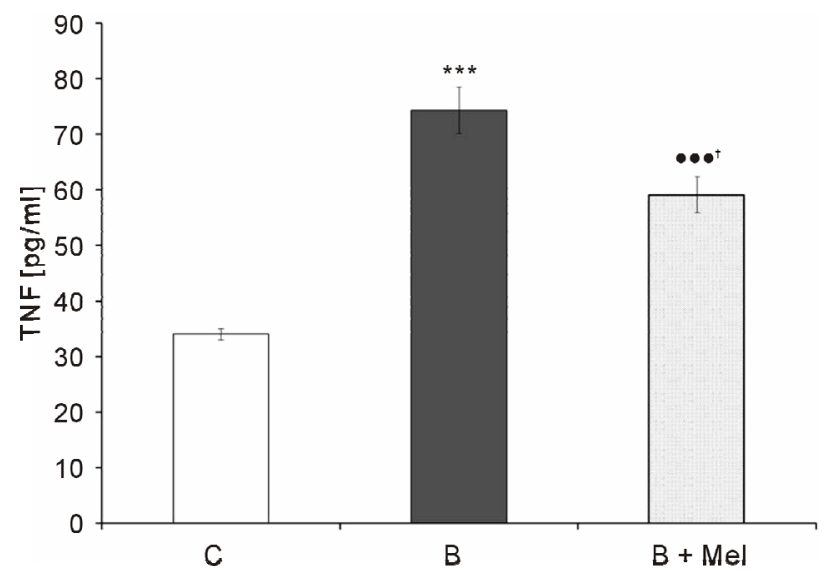

Figure 2. Effect of melatonin on burn-induced hepatic TNFalpha level. Results are given as mean \pm SEM; ${ }^{* * *}$ p $<0.001$ compared with Control group; $\bullet \bullet p<0.001$ compared with burned group. increased in sinusoidal endothelial cells of the burned group treated with melatonin (Figure 3). It was moderate to strong in sinusoidal endothelial cells and their mean content $(2.22 \pm 0.74)$ was significantly higher $63 \%$ (p < $0.001)$ than this of the controls.

\subsection{Changes in the Expression on Apoptosis-Related Bax in Liver}

The data showed that Bax expression was detected mainly in the SECs in the control group (Figure 4). The intensity of the immune reaction ranged from mild to moderate (1.46 \pm 0.29$)$ Bax expression in SECs and isolated hepatocytes was moderate to strong $(2.34 \pm 0.59)$ in the burned goup and the number of positive cells was significantly higher by $60 \%(\mathrm{p}<0.001)$ compared to controls. The expression Bax was reduced by $62 \%$ (p < 0.01 ) in the burned group treated with melatonin maintaining the level close to the control values.

\subsection{Changes in the Ratio Bax/Bcl-2 in Liver}

The results presented in Table 1 showed that the ratio BAX/Bcl-2 was significantly higher by $91 \%(\mathrm{p}<0.01)$ in the burned group compared to controls. Treatment with melatonin significantly reduced the $\mathrm{BAX} / \mathrm{Bcl}-2$ ratio after burns maintaining the level close to the control values.

\section{DISCUSSION}

Burn trauma initiates systemic inflammatory response where the generation of proinflammatory cytokines and free radicals causes tissue injury to skin and to remote organs liver including [4-6,20]. A significant increase of proinflammatory cytokine TNF- $\alpha$ and MDA as a marker of lipid peroxidation in liver has been reported in our animal models of burns along with evidence of histopathological changes such as degenerative changes and hepatic apoptotic bodies (Figure 5) occurred in rats after burns. The present findings showed that administration of antioxidant melatonin can block this progress by attenuating oxidative stress, inflammatory response and apoptosis of SECs and hepatocytes.

Table 1. Changes in the expression of Bax, Bcl-2 in liver tissue of rats after burns and ratio of $\mathrm{Bax} / \mathrm{Bcl}-2$.

\begin{tabular}{cccc}
\hline Group & Bcl-2 & Bax & Bax/Bcl-2 \\
\hline C group & $1.36 \pm 0.048$ & $1.46 \pm 0.25$ & 1.07 \\
B group & $1.14 \pm 0.69$ & $2.34 \pm 0.59^{* * *}$ & $2.05^{*}$ \\
B+MEL group & $2.22 \pm 0.74^{+++}$ & $1.46 \pm 0.29^{+}$ & $1.23^{+}$ \\
\hline
\end{tabular}

Results are given as mean $\pm \mathrm{SEM} ;{ }^{* * *} \mathrm{p}<0.001$ compared with Control (C) group; ${ }^{+++} \mathrm{p}<0.001$ compared with burned (B) group. ${ }^{*} \mathrm{p}<0.05$ compared with Control (C) group; ${ }^{+} \mathrm{p}<0.05$ compared with burned (B) group. 

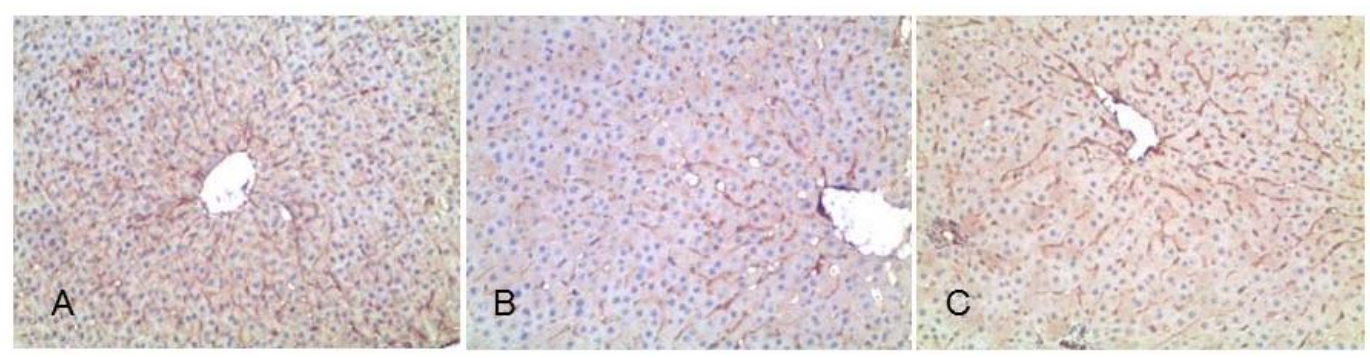

Figure 3. Immunohistochemistry detection for Bcl-2 in liver after burns. It is localized in sinusoidal endothelial cells (SECs) in the control group (A). In the burned group, Bcl-2 expression remained low (B) The expression $\mathrm{Bcl}-2$ of the burned group treated with melatonin was moderate to strong in SECs $(\mathrm{C})$.

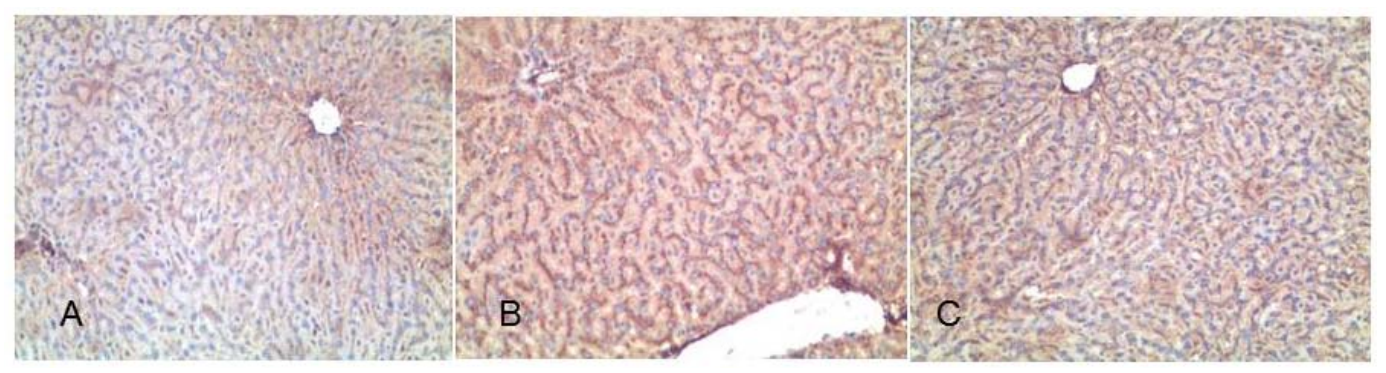

Figure 4. Immunohistochemistry detection for Bax in liver after burns. Original magnification, $200 \times$. It is localized in sinusoidal endothelial cells (SECs) of the liver in the control group. (A) The staining intensity of Bax positive cells was mild to moderate. In the burned group induction of Bax positive cells was principally in SECs and isolated hepatocytes around the central vein (B). It was moderate to strong in the individually cells. The expression BAX was reduced in the burned group treated with melatonin maintaining the level close to the control values (C).

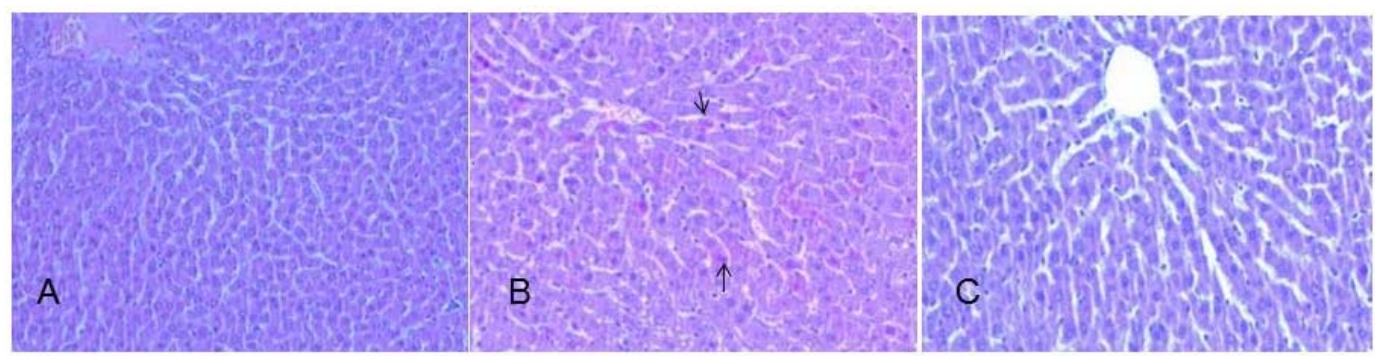

Figure 5. Effect of melatonin on burn-induced histomorphological changes in liver in rats (A) control group, (B) burned group, (C) burned, treated with melatonin group. Original magnification $400 \times$. H \& E staining, original magnification. Arrows indicate apoptotic cells.

Oxidative stress is recognized as an important mechanism of apoptosis [21,22]. The involvement of ROS is suggested in apoptotic cell death of hepatocytes $[8,23]$ and endothelial cells [23].

Mitochondria are an important source and target of ROS and RNS even when their production is growing at a reduced antioxidant protection. ROS and RNS activate lipid peroxidation damage and mitochondrial membranes, protein and DNA. During the ROS-induced lipid peroxidation and damage of mtDNA of the cell (internal signals) pro-apoptotic Bax which underwent a conformational change (externalization of phosphatidylserine (ps) translocates from the cytosol to the mitochondrial membrane where anti-apototic proteins Bcl-2 are localized [11]. The
Bcl-2 prevents the cell death whereas Bax forming heterodimers with Bcl-2 appears to accelerate the cell death signal [24]. The increased ROS production results in an imbalance between anti-and pro-apoptotic Bcl-2 family proteins, membrane permeability transition, release of cytochome $\mathrm{C}$ and further production of ROS, which contributes to the susceptibility of the cell to apoptosis [25].

In the current study, we observed a significant increase in Bax protein levels in SECs at the periphery of the sinusoidal space in liver. Burn-induced lipid peroxidation was found to be accompanied by elevation of pro-apoptotic Bax protein without changes of anti-apoptotic Bcl-2 protein of burn-treated animals compared with the control group animals. This was confirmed by the ratio Bax/ 
Bcl-2 which was higher in the burned group compared with the control group and the large number of Bax possitive stained SECs and hepatocytes together with few Bcl-2 possitive stained SEC and hepatocytes (Table 1) indicating the susceptibility of these cells to apoptosis.

Our results were consistent with privious reports about elevation of Bax protein levels and reduction of the anti-apoptotic protein $\mathrm{Bcl}-2$ and its translocation to mitochondria causing apoptosis in the gut after experimenttal thermal trauma [26]. Similar findings for apoptotic proteins Bax and Bcl-2 in SECs and hepatocytes are demonstrated in cold I/R model [27].

The expression of proapototic BAX protein can also be increased and under the action of external death signals $[28,29]$. The increased Bax proapoptotic protein expression may associate with translocation of proapoptotic Bid protein to mitochondria by the Fas/TNF-R1 pathway [30]. There is strong evidence that burn induces Fas/FasL [31] representing one possible pathway for burn induction of hepatic apoptosis and dysfunction. Bid-mediated mitochondria pathway seems to be critical in certain types of cells, such as hepatocytes [32]. Thus, Bid connects the death receptor pathway and the mitochondria pathway and is responsible for cytochrome $\mathrm{C}$ release and the downstream caspase activities after Fas/ TNF-R1 activation. Overexpression of pro-inflamematory cytokines and decreased level of $\mathrm{Bcl}-2$ protein were reported in liver after burns [26]. Jeschke et al. (2001) investigating molecular mechanisms of burn-induced apoptosis and dysfunction found out that burn injury leads to endoplasmic reticulum (ER) stress, mitochondrial damage with release of cytochome $\mathrm{C}$ and apoptotic cell death. Other authors suggest that Bid can induce release of cytochrome $\mathrm{C}$ by mechanism independent of Bax and increasing permeability of the mitochondrial membrane [16].

Treatment of the animals with melatonin $(2 \times 10 \mathrm{mg} / \mathrm{kg})$ significantly prevented those changes resulting in a significantly lower ratio $\mathrm{Bax} / \mathrm{Bcl}-2$ compared with burned untreated rats. Melatonin is able to shift the balance towards a cell-protective state. Indeed many studies demonstrate that antiapoptotic effect involves the down regulation of BAX or up regulation of Bcl-2 [11]. Melatonin increased expression of Bcl-2, decreased expression of Bax in SECs and hepatocytes, indicating decreased susceptibility of these cells to apoptosis and hepatic damage in burned rats. Therefore, anti-apoptotic effects of melatonin are related with decrease of ROS production in mitochondria and activate of anti-apoptotic and redox-sensitive system Bcl-2/Bax.

The present study provides morphological and molecular biological evidence for the protective role of the melatonin in ameliorating oxidative stress, hepatic apoptosis, and inflammation in experimental thermal trauma.
Melatonin may protect liver from oxidative stress by scavenging the free radicals in mitochondria inhibiting lipid peroxidation and production of proinflammatory cytokine TNF- $\alpha$. Thus, melatonin modulates the expression of Bcl-2 family proteins by increasing anti-apoptotic Bcl-2 in SECs and by inhibiting apoptosis restricts the damage of the liver.

\section{REFERENCES}

[1] Lausevic, Z., Lausevic, M., Trbojevic-Stankovic, B., Krstic, S. and Stojimirovic, B. (2008) Predicting multiple organ failure in patients with severe trauma. Canadian Journal of Surgery, 51, 97-102.

[2] Jeschke, M.G. (2009) The hepatic response to thermal injury: Is the liver important for postburn outcomes. Molecular Medicine, 15, 337-351.

http://dx.doi.org/10.2119/molmed.2009.00005

[3] Nishiura, T., Nishimura, T., de Serres, S., Godfrey, V., Bradham, C.A., Nakagawa, T., Brenner, D.A. and Meyer, A.A. (2000) Gene expression and cytokine and enzyme activation in the liver after a burn injury. Journal of Burn Care \& Rehabilitation, 21, 135-141. http://dx.doi.org/10.1097/00004630-200021020-00009

[4] Chen, X.L., Xia, Z.F., Yu, Y.X., Wei, D., Wang, C.R. and Ben, D.F. (2005) p38 mitogen-activated protein kinase inhibition attenuates burn-induced liver injury in rats. Burns, 31, 320-330.

http://dx.doi.org/10.1016/j.burns.2004.10.015

[5] Gauglitz, G.G., Song, J., Herndon, D.N., Finnerty, C.C., Boehning, D., Barral, J.M. and Jeschke, M.G. (2008) Characterization of the inflammatory response during acute and post-acute phases after severe burn. Shock, 30, 503-507. http://dx.doi.org/10.1097/SHK.0b013e31816e3373

[6] Agay, D., Andriollo-Sanchez, M., Claeyssen, R., Touvard, L., Denis, J., Roussel, A.M. and Chancerelle, Y. (2008) Interleukin-6, TNF-alpha and interleukin-1 beta levels in blood and tissue in severely burned rats. European Cytokine Network, 19, 1-7.

[7] Gravante, G., Delogu, D. and Sconocchia, G. (2007) "Systemic apoptotic response" after thermal burns. Apoptosis, 12, 259-270. http://dx.doi.org/10.1007/s10495-006-0621-8

[8] Kurose, I., Higuchi, H., Miura, S., Saito, H., Watanabe, N., Hokari, R., Hirokawa, M., Takaishi, M., Zeki, S., Nakamura, T., Ebinuma, H., Kato, S. and Ishii, H. (1997) Oxidative stress-mediated apoptosis of hepatocytes exposed to acute ethanol intoxication.Hepatology, 25, 368-378. http://dx.doi.org/10.1002/hep.510250219

[9] Guha, M., Maity, P., Choubey, V., Mitra, K., Reiter, R.J. and Bandyopadhyay, U. (2007) Melatonin inhibits free radical-mediated mitochondrial-dependent hepatocyte apoptosis andliver damage induced during malarial infection. Journal of Pineal Research, 43, 72-81. http://dx.doi.org/10.1111/j.1600-079X.2007.00488.x

[10] Maity, P., Bindu, S., Dey, S., Goyal, M., Alam, A., Pal, C., Reiter, R. and Bandyopadhyay, U. (2009) Melatonin 
reduces indomethacin-induced gastric mucosal cell apoptosis by preventing mitochondrial oxidative stress and the activation of mitochondrial pathway of apoptosis. Journal of Pineal Research, 46, 314-323. http://dx.doi.org/10.1111/j.1600-079X.2009.00663.x

[11] Reiter, R.J., Tan, D.X., Manchester, L.C. and Qi, W. (2001) Biochemical reactivity of melatonin with reactive oxygen and nitrogen species: A review of the evidence. Cell Biochemistry and Biophysics, 34, 237-256. http://dx.doi.org/10.1385/CBB:34:2:237

[12] Reiter, R.J. (2000) Melatonin. Lowering the high price of free radicals. News in Physiological Sciences, 15, 246250.

[13] Rodriguez, C., Mayo, J.C., Sainz, R.M., Antolnn, I., Herrera, F., Marthn, V. and Reiter, R.J. (2004) Regulation of antioxidant enzymes: A significant role for melatonin. Journal of Pineal Research, 36, 1-9. http://dx.doi.org/10.1046/j.1600-079X.2003.00092.x

[14] López, A., García, J.A., Escames, G., Venegas, C., Ortiz, F., López, L.C. and Acuña-Castroviejo, D. (2009) Melatonin protects the mitochondria from oxidative damage reducing oxygen consumption, membrane potential, and superoxide anion production. Journal of Pineal Research, 46, 188-198. http://dx.doi.org/10.1111/j.1600-079X.2008.00647.x

[15] Acuña Castroviejo, D., López, L.C., Escames, G., López, A., García, J.A. and Reiter, R.J. (2011) Melatonin-mitochondria interplay in health and disease. Current Topics in Medicinal Chemistry, 11, 221-240.

[16] Kim, S.H. and Lee, S.M. (2011) Cytoprotective effects of melatonin against necrosis and apoptosis induced by ischemia/reperfusion injury in rat liver. Journal of Pineal Research, 44, 165-171. http://dx.doi.org/10.1111/j.1600-079X.2007.00504.x

[17] Tuñón, M.J., San Miguel, B., Crespo, I., Jorquera, F., Santamaría, E., Alvarez, M., Prieto, J. and GonzálezGallego, J. (2011) Melatonin attenuates apoptotic liver damage in fulminant hepatic failure induced by the rabbit hemorrhagic disease virus. Journal of Pineal Research, 50, 38-48.

http://dx.doi.org/10.1111/j.1600-079X.2010.00807.x

[18] Porter, N.A., Nixon, J.R. and Isaac, R. (1976) Cyclic peroxidase and thiobarbituric assay. Biochimica et Biophysica Acta, 441, 596-599.

[19] Tzaneva, M. (2001) Electron microscopic immunohistochemical investigation of chromogranin A in endocrine cells in human oxyntic gastric mucosa. Acta Histochemica, 103, 179-194. http://dx.doi.org/10.1078/0065-1281-00585

[20] Jeschke, M.G., Einspanier, R., Klein, D. and Jauch, K.W. (2002) Insulin attenuates the systemic inflammatory response to thermal trauma. Molecular Medicine, 8, 443450 .

[21] Sarafian, T.A. and Bredesen, D.E. (1994) Is apoptosis mediated by reactive oxygen species? Free Radical Research, 21, 1-8.

http://dx.doi.org/10.3109/10715769409056549

[22] Singh, R. and Czaja, M.J. (2007) Regulation of hepatocyte apoptosis by oxidative stress. Journal of Gastroenterology and Hepatology, 22, S45-S48. http://dx.doi.org/10.1111/j.1440-1746.2006.04646.x

[23] Rauen, U., Polzar, B., Stephan, H., Mannherz, H.G. and de Groot, H. (1999) Cold-induced apoptosis in cultured hepatocytes and liver endothelial cells: Mediation by reactive oxygen species. FASEB Journal, 13, 155-168.

[24] Radogna, F., Diederich, M. and Ghibelli, L. (2010) Melatonin: A pleiotropic molecule regulating inflammation. Biochemical Pharmacology, 80, 1844-1852. http://dx.doi.org/10.1016/j.bcp.2010.07.041

[25] Lucken-Ardjomande, S. and Martinou, J.C. (2005) Regulation of Bcl-2 proteins and of the permeability of the outer mitochondrial membrane. Comptes Rendus Biologies, 328, 616-631. http://dx.doi.org/10.1016/j.crvi.2005.05.002

[26] Klein, D., Schubert, T., Horch, R.E., Jauch, K.W. and Jeschke, M.G. (2004) Insulin treatment improves hepatic morphology and function through modulation of hepatic signals after severe trauma. Annals of Surgery, 240, 340349. http://dx.doi.org/10.1097/01.sla.0000133353.57674.cd

[27] Yue, L.H., Zhao, Y.L., Chen, J. and Lu, D.R. (2010) Effect of fusion protein TAT and heme oxygenase-1 on liver sinusoidal endothelial cells apoptosis during preservation injury. Chinese Medical Journal, 123, 68-73.

[28] Ding, W.X. and Yin, X.M. (2004) Dissection of the multiple mechanisms of TNF-alpha-induced apoptosis in liver injury. Journal of Cellular and Molecular Medicine, 8, 445-454. http://dx.doi.org/10.1111/j.1582-4934.2004.tb00469.x

[29] Shuh, M., Bohorquez, H., Loss Jr., G.E. and Cohen, A.J. (2013) Tumor necrosis factor- $\alpha$ : Life and death of hepatocytes during liver ischemia/reperfusion injury. Ochsner Journal, 13, 119-130.

[30] Yin, XM. (2000) Bid, a critical mediator for apoptosis induced by the activation of Fas/TNF-R1 death receptors in hepatocytes. Journal of Molecular Medicine, 78, 203211. http://dx.doi.org/10.1007/s001090000099

[31] Jeschke, M.G., Micak, R.P., Finnerty, C.C. and Herndon, D.N. (2007) Changes in liver function and size after a severe thermal injury. Shock, 28, 172-177. http://dx.doi.org/10.1097/shk.0b013e318047b9e2

[32] Chen, X., Ding, W.X., Ni, H.M., Gao, W., Shi, Y.H., Gambotto, A.A., Fan, J., Beg, A.A. and Yin, X.M. (2007) Bid-independent mitochondrial activation in tumor necrosis factor alpha-induced apoptosis and liver injury. Molecular and Cellular Biology, 27, 541-553. http://dx.doi.org/10.1128/MCB.01166-06 


\section{ABBREVIATIONS}

SECs-Sinusoidal Endothelial Cells,

ROS-Reactive Oxygen Species,

RNS-Reactive Nitrogen Species,

MDA—Malondialdehyde,
TNF- $\alpha$ - Tumor Necrosis Factor, Bcl-2-Anti-Apoptotic Protein, Bax-Pro-Apoptotic Protein, $\mathrm{I} / \mathrm{R}$-Ischemia/Reperfusion, TBSA-Total Body Surface Area. 